\title{
Four exact relations for the effective relaxation function of linear viscoelastic composites
}

\author{
P. Suquet ${ }^{\mathrm{a}}$ \\ ${ }^{\mathrm{a}}$ LMA, CNRS, UPR 7051, Aix-Marseille Univ., Centrale Marseille, F-13402 Marseille Cedex 20, France
}

\begin{abstract}
This study is devoted to viscoelastic composites composed from individual Maxwell constituents. The effective constitutive relations of such composites exhibit a long memory effect which manifests itself through an integral kernel (the effective relaxation function of the composite). Four asymptotic relations for this integral kernel are derived which require only the resolution of linear elastic (or purely viscous) problems. These four relations can be used in an approximate model with two relaxation times (for incompressible, isotropic composites). The model is exact for specific microstructures but is an approximation in general. Its accuracy is discussed by comparison with full-field simulations.
\end{abstract}

To cite this article: P. Suquet, C. R. Mécanique xxx (2011).

\section{Résumé}

Quatre relations exactes pour la fonction de relaxation effective de composites viscoélastiques linéaires. Cette étude est consacrée à l'étude du comportement effectif de composites dont les constituants élémentaires sont des matériaux viscoélastiques de Maxwell. Ce comportement effectif, à mémoire longue, s'exprime à l'aide d'une fonction de relaxation effective. Nous établissons que cette fonction de relaxation satisfait quatre relations qui peuvent être exprimées par des calculs ne faisant intervenir que des problèmes de type élasticité linéaire. Ces quatre relations peuvent être utilisées pour proposer un modèle approché du comportement effectif à deux temps de relaxation. L'analyse détaillée de deux exemples montre que ce modèle est exact dans un cas, et constitue une bonne approximation du comportement effectif dans le second cas.

Email address: suquet@lma.cnrs-mrs.fr (P. Suquet). 


\section{Introduction}

This study is devoted to the effective behavior of composites made from linear viscoelastic phases, more specifically Maxwellian phases which are characterized by a relation between the linearized strain $\varepsilon$ and the Cauchy stress $\boldsymbol{\sigma}$ in the form:

$$
\dot{\varepsilon}=M_{e}^{(r)}: \dot{\sigma}+M_{v}^{(r)}: \sigma,
$$

where $\boldsymbol{M}_{e}^{(r)}$ and $\boldsymbol{M}_{v}^{(r)}$ denote respectively the elastic and viscous compliance moduli of the phase and where an overdot denotes derivation with respect to time.

As is well known, even when the individual phases have a "short memory" (such as given by the Maxwell model (1)), the effective behavior of composites made from different such phases may exhibit a "long memory" effect which manifests itself in the effective constitutive relations through an integral kernel (Sanchez and Sanchez [1], Laws and Mc Laughlin [2], Suquet [3], Francfort and Suquet [4], Turner and Tomé [5], Rougier, Stolz and Zaoui [6] among others):

$$
\overline{\boldsymbol{\sigma}}(t)=\frac{d}{d t}\left(\int_{0}^{t} \widetilde{\boldsymbol{L}}(t-s): \overline{\boldsymbol{\varepsilon}}(s) d s\right) .
$$

where an overall bar denotes spatial averaging over a representative volume element. $\widetilde{\boldsymbol{L}}(t)$ is the effective relaxation function of the composite.

The aim of this study is to derive a set of four tensorial relations which must be satisfied by $\widetilde{\boldsymbol{L}}(t)$ for small or large time $t$, or equivalently by its Laplace transform $\widetilde{\boldsymbol{L}}^{*}(p)$ for small and large $p$ involving only quantities which can be evaluated by solving linear elastic problems. In particular, these relations provide restrictions on models which approximate the integral kernel $\widetilde{\boldsymbol{L}}(t)$ when it is not explicitly known. A common practice, called the collocation method, consists in approximating $\widetilde{\boldsymbol{L}}(t)$ by a Prony series with a finite number of relaxation times, each of them corresponding to an exponentially decreasing relaxation function:

$$
\widetilde{\boldsymbol{L}}(t) \simeq \sum_{i=1}^{M} \overline{\boldsymbol{L}}_{i} e^{-t / \bar{\tau}_{i}} .
$$

The four relations derived in section 3 impose restrictions on the tensors $\overline{\boldsymbol{L}}_{i}$ and on the relaxation times $\bar{\tau}_{i}$. An approximate model based on these relations and involving 2 relaxation times for incompressible isotropic composites and 4 for compressible isotropic composites is proposed in section 3.3 and the accuracy of this model is discussed in section 4 . This model is in fact exact in the specific case of incompressible particle-matrix composites having a microstructure such the Hashin-Shtrikman's estimate is exact for the effective linear elastic properties, but is only an approximation for other microstructures.

\section{Maxwellian composites}

\subsection{Individual phases, local problem and effective behavior}

The composite materials considered in the present study are made from $N$ different homogeneous constituents, or phases, which are assumed to be randomly distributed in a specimen occupying a volume $V$, at a length scale that is much smaller than the size of $V$. Each constituent is linear viscoelastic (and Maxwellian) governed by the constitutive relations (1) and characterized by elastic and viscous compliances $\boldsymbol{M}_{e}^{(r)}$ and $\boldsymbol{M}_{v}^{(r)}$ (with inverse $\boldsymbol{L}_{e}^{(r)}$ and $\boldsymbol{L}_{v}^{(r)}$ ). Define 


$$
\boldsymbol{M}_{e}(\boldsymbol{x})=\sum_{r=1}^{N} \boldsymbol{M}_{e}^{(r)} \chi^{(r)}(\boldsymbol{x})
$$

(with a similar definition for $\boldsymbol{M}_{v}(\boldsymbol{x})$ ), where $\chi^{(r)}$ is the characteristic function of phase $r$. The volume averages of a function $f$ over the composite $V$ and over phase $\mathrm{r}$ are denoted as $\bar{f}$ and $\bar{f}^{(r)}$ respectively.

The local problem to be solved to determine the local stress and strain fields in the volume element $V$ consists of the equilibrium equations, compatibility conditions, constitutive relations and boundary conditions ${ }^{1}$.

$$
\begin{array}{lr}
\dot{\boldsymbol{\varepsilon}}(\boldsymbol{x}, t)=M_{e}(\boldsymbol{x}): \dot{\boldsymbol{\sigma}}(\boldsymbol{x}, t)+\boldsymbol{M}_{v}(\boldsymbol{x}): \boldsymbol{\sigma}(\boldsymbol{x}, t), & \text { for }(\boldsymbol{x}, t) \in V \times[0, T], \\
\operatorname{div} \boldsymbol{\sigma}=0 & \text { for }(\boldsymbol{x}, t) \in V \times[0, T], \\
\langle\varepsilon(t)\rangle=\overline{\boldsymbol{\varepsilon}}(t)+\text { boundary conditions on } \partial V . &
\end{array}
$$

The effective behavior of the composite is defined as the relation between the average stress $\overline{\boldsymbol{\sigma}}(t)$ at time $t$ and the history of the average strain before $t, \bar{\varepsilon}(s), 0 \leq s \leq t$.

\subsection{Local problem in Laplace-Carson space}

A common practice in the study of linearly viscoelastic systems is to transform the evolution equations (4) into an elastic problem by means of the Laplace-Carson (LC) transform (see appendix A for the definition and a few useful properties of the LC transform). Let us denote the LC transform of a function $f(t)$ as $f^{*}(p)$. Then the local problem (4) becomes:

$$
\left.\begin{array}{lr}
\varepsilon^{*}(\boldsymbol{x}, p)=\left(\boldsymbol{M}_{e}(\boldsymbol{x})+\frac{1}{p} \boldsymbol{M}_{v}(\boldsymbol{x})\right): \boldsymbol{\sigma}^{*}(\boldsymbol{x}, p)+\varepsilon_{0}(\boldsymbol{x})-\boldsymbol{M}_{e}(\boldsymbol{x}): \boldsymbol{\sigma}_{0}(\boldsymbol{x}), & \text { for } \boldsymbol{x} \in V \\
\operatorname{div}\left(\boldsymbol{\sigma}^{*}(\boldsymbol{x}, p)\right)=0, & \text { for } \boldsymbol{x} \in V \\
\left\langle\varepsilon^{*}(p)\right\rangle=\overline{\boldsymbol{\varepsilon}}^{*}(p)+\text { boundary conditions on } \partial V . &
\end{array}\right\}
$$

where $\boldsymbol{\varepsilon}^{*}(p)$ and $\boldsymbol{\sigma}^{*}(p)$ are the LC transforms of $\boldsymbol{\varepsilon}$ and $\boldsymbol{\sigma}$ while $\boldsymbol{\varepsilon}_{0}(\boldsymbol{x})$ and $\boldsymbol{\sigma}_{0}(\boldsymbol{x})$ denote the initial values of the local fields $\varepsilon$ and $\boldsymbol{\sigma}$. The "eigenstrain" $\varepsilon_{0}-\boldsymbol{M}_{e}: \boldsymbol{\sigma}_{0}$ is the initial viscous strain. For simplicity, the initial state of the composite will be assumed to be purely elastic, or in other words:

$$
\varepsilon_{0}(\boldsymbol{x})-\boldsymbol{M}_{e}(\boldsymbol{x}): \boldsymbol{\sigma}_{0}(\boldsymbol{x})=0 \text { for all } \boldsymbol{x} \in V .
$$

The constitutive equations in Laplace space can be given two equivalent forms reminiscent either of a linear elastic problem or of a linearly viscous problem. The first writing of (5) relates $\boldsymbol{\sigma}^{*}(p)$ to $\boldsymbol{\varepsilon}^{*}(p)$ :

$$
\left.\begin{array}{rl} 
& \boldsymbol{\sigma}^{*}(p)=\boldsymbol{L}^{(r)^{*}}(p): \boldsymbol{\varepsilon}^{*}(p) \quad \text { in phase } r, \\
= & \left(\boldsymbol{M}^{(r)^{*}}(p)\right)^{-1}, \quad \boldsymbol{M}^{(r)}(p)=\boldsymbol{M}_{e}^{(r)}+\frac{1}{p} \boldsymbol{M}_{v}^{(r)},
\end{array}\right\}
$$

The effective behavior of the composite in Laplace space is characterized by an effective "stiffness" tensor $\widetilde{\boldsymbol{L}}^{*}(p)$

$$
\overline{\boldsymbol{\sigma}}^{*}(p)=\widetilde{\boldsymbol{L}}^{*}(p): \overline{\boldsymbol{\varepsilon}}^{*}(p) .
$$

1. Boundary conditions are not specified and discussed here. They are of the classical types, see Suquet [3] or Ponte Castañeda and Suquet [10] for more details 
The effective relaxation function $\widetilde{\boldsymbol{L}}(t)$ entering (2) is the inverse LC transform of $\widetilde{\boldsymbol{L}}^{*}(p)$.

The alternative writing of (5) relates $\boldsymbol{\sigma}^{*}(p)$ to $\boldsymbol{\varepsilon}^{\prime *}(p)=p \boldsymbol{\varepsilon}^{*}(p)$ which is the LC transform of $\dot{\boldsymbol{\varepsilon}}$ :

$$
\boldsymbol{\sigma}^{*}(p)=\mathcal{L}^{(r)^{*}}(p): \boldsymbol{\varepsilon}^{\prime *}(p), \quad \mathcal{L}^{(r)^{*}}(p)=\frac{1}{p} \boldsymbol{L}^{(r)^{*}}(p)=\left(p \boldsymbol{M}_{e}^{(r)}+\boldsymbol{M}_{v}^{(r)}\right)^{-1}
$$

and the effective constitutive relations in Laplace space, equivalent to (7), read as:

$$
\overline{\boldsymbol{\sigma}}^{*}(p)=\widetilde{\mathcal{L}}^{*}(p): \overline{\boldsymbol{\varepsilon}}^{* *}(p), \quad \widetilde{\mathcal{L}}^{*}(p)=\frac{1}{p} \widetilde{\boldsymbol{L}}^{*}(p) .
$$

According to relation (A.2), $\widetilde{\mathcal{L}}^{*}(p)$ is the LC transform of

$$
\widetilde{\mathcal{L}}(t)=\int_{0}^{t} \widetilde{\boldsymbol{L}}(s) d s
$$

The effective tensors $\widetilde{\boldsymbol{L}}^{*}(p)$ and $\widetilde{\mathcal{L}}^{*}(p)$ can be given explicit forms for specific microstructures. These expressions will be specified in section 4 .

\subsection{A Maxwellian approximation to the effective behavior of viscoelastic composites}

Given the constitutive relations (1) of the individual phases, it is tempting to approximate the exact effective behavior of the composite by a Maxwellian relation in the form:

$$
\dot{\bar{\varepsilon}}=\widetilde{\boldsymbol{M}}_{e}: \dot{\bar{\sigma}}+\widetilde{\boldsymbol{M}}_{v}: \overline{\boldsymbol{\sigma}} .
$$

A nice feature of this relation is that it requires only the evaluation of two effective tensors, the effective stiffness $\widetilde{\boldsymbol{L}}_{e}$ and the effective viscosity $\widetilde{\boldsymbol{L}}_{v}$ which can can be obtained by standard "elastic" homogenization procedures. Unfortunately, it is only an approximation to the actual effective behavior of the composite, which gives accurate prediction only for small and large $t$ as is well-known (and will be illustrated below).

Indeed the approximation corresponding to (11) in Laplace space amounts to considering that the effective elastic moduli for a linear composite with elastic moduli $\boldsymbol{L}^{(r)^{*}}(p)$ (or equivalently $\mathcal{L}^{(r)^{*}}(p)$ ) would be:

$$
\widetilde{\boldsymbol{L}}_{\text {Maxw }}^{*}(p)=\left(\widetilde{\boldsymbol{M}}_{e}+\frac{1}{p} \widetilde{\boldsymbol{M}}_{v}\right)^{-1}, \quad \widetilde{\mathcal{L}}_{\text {Maxw }}^{*}(p)=\left(p \widetilde{\boldsymbol{M}}_{e}+\widetilde{\boldsymbol{M}}_{v}\right)^{-1}
$$

This is not true, as the homogenized (or effective) tensor of a sum of tensors is not the sum of the homogenized tensors.

However the approximation (11) is (asymptotically) exact for small and large time $t$, or equivalently the approximation (12) is exact in Laplace space for small and large $p$. It is indeed straightforward to note that:

$$
\lim _{p \rightarrow+\infty} \widetilde{\boldsymbol{L}}_{M a x w}^{*}(p)=\widetilde{\boldsymbol{L}}_{e}, \quad \lim _{p \rightarrow 0} \widetilde{\mathcal{L}}_{\text {Maxw }}^{*}(p)=\widetilde{\boldsymbol{L}}_{v} .
$$

Although the exact $\widetilde{\boldsymbol{L}}^{*}(p)$ and $\widetilde{\mathcal{L}}^{*}(p)$ are not given by (12), they do satisfy the above asymptotic limits (13) which are the first two relations given below. Two additional asymptotic relations for the derivatives with respect to $p$ of $\widetilde{\boldsymbol{L}}^{*}(p)$ and $\widetilde{\mathcal{L}}^{*}(p)$ are also derived. 


\section{Four relations for the effective relaxation function}

The aim of this section is to study the asymptotic behavior of the relaxation function $\widetilde{\boldsymbol{L}}(t)$ as $t$ tends to 0 or $+\infty$, or equivalently of its LC transform $\widetilde{\boldsymbol{L}}^{*}(p)$ as $p$ tends to $+\infty$ or 0 (according to (A.4) and (A.5) the asymptotic behaviors of a function and of its Laplace transform are related).

$$
\begin{gathered}
\lim _{p \rightarrow 0} \widetilde{\mathcal{L}}^{*}(p)=\widetilde{\boldsymbol{L}}_{v} . \\
\lim _{p \rightarrow+\infty} \widetilde{\boldsymbol{L}}^{*}(p)=\widetilde{\boldsymbol{L}}_{e} . \\
\lim _{p \rightarrow 0} \overline{\boldsymbol{\varepsilon}}^{\prime}: \frac{\partial \widetilde{\mathcal{L}}^{*}}{\partial p}(p): \overline{\boldsymbol{\varepsilon}}^{\prime}=-\left\langle\boldsymbol{\sigma}_{v}: \boldsymbol{M}_{e}: \boldsymbol{\sigma}_{v}\right\rangle, \\
\lim _{p \rightarrow+\infty}\left(p^{2} \overline{\boldsymbol{\varepsilon}}: \frac{\partial \widetilde{\boldsymbol{L}}^{*}}{\partial p}(p): \overline{\boldsymbol{\varepsilon}}\right)=\left\langle\boldsymbol{\sigma}_{e}: \boldsymbol{M}_{v}: \boldsymbol{\sigma}_{e}\right\rangle,
\end{gathered}
$$

where $\boldsymbol{\sigma}_{v}$ is the solution is the local stress field solution of the purely viscous problem (linearly viscous composite with the same geometry as the actual one and viscosity moduli $\boldsymbol{L}_{v}^{(r)}$ in phase $r$ ) for a macroscopic strain-rate $\overline{\boldsymbol{\varepsilon}}^{\prime} . \widetilde{\boldsymbol{L}}_{v}$ is the effective viscosity tensor of this composite. Similarly $\boldsymbol{\sigma}_{e}$ is the solution is the local stress field solution of the purely elastic problem (linearly elastic composite with the same geometry as the actual one and elastic stiffness $\boldsymbol{L}_{e}^{(r)}$ in phase $r$ ) for a macroscopic deformation $\bar{\varepsilon}$. $\widetilde{\boldsymbol{L}}_{e}$ is the effective stiffness of this composite.

Note that these 4 relations make use of only the solution of linear elastic (or purely viscous) problems.

\subsection{Interpretation}

Before proceeding to the interpretation of the first two relations, let us consider first a single Maxwellian material. When it is deformed at constant strain-rate from an initial undeformed and unstressed state, its initial response (for small $t$ ) is governed by its elastic stiffness $\boldsymbol{L}_{e}$ whereas its large time behavior is governed its viscous tensor $\boldsymbol{L}_{v}$. This can be seen from (1) by noting that for small $t, \boldsymbol{\sigma}(t)$ is small and the constitutive relation (1) reduces to $\dot{\boldsymbol{\sigma}} \simeq \boldsymbol{L}_{e}: \dot{\boldsymbol{\varepsilon}}$. In the other limit, as $t$ becomes large, the stress approaches a stationary value (bearing in mind that the strain-rate is kept constant), the stress-rate $\dot{\boldsymbol{\sigma}}$ vanishes and the constitutive relation (1) reduces to $\boldsymbol{\sigma} \simeq \boldsymbol{L}_{v}: \dot{\varepsilon}$.

A similar interpretation holds at the composite level: (15) states that the instantaneous response of the composite (for small $t$, or equivalently large $p$ ) is governed by the homogenized elastic moduli of the phases, whereas, according to (14), its long time behavior (for large $t$, or equivalently small $p$ ) is governed by its purely viscous effective tensor.

Unfortunately, there is no similar explanation for the other two relations (16) and (17). They involve the second moments per phase of the asymptotic stress fields (for small and large $t$ ):

$$
\left\langle\boldsymbol{\sigma}_{e}: \boldsymbol{M}_{v}: \boldsymbol{\sigma}_{e}\right\rangle=\sum_{r=1}^{N} c^{(r)} \boldsymbol{M}_{v}^{(r)}::\left\langle\boldsymbol{\sigma}_{e} \otimes \boldsymbol{\sigma}_{e}\right\rangle^{(r)}, \quad\left\langle\boldsymbol{\sigma}_{v}: \boldsymbol{M}_{e}: \boldsymbol{\sigma}_{v}\right\rangle=\sum_{r=1}^{N} c^{(r)} \boldsymbol{M}_{e}^{(r)}::\left\langle\boldsymbol{\sigma}_{v} \otimes \boldsymbol{\sigma}_{v}\right\rangle^{(r)} .
$$

The second moments per phase of the stress fields $\boldsymbol{\sigma}_{e}$ and $\boldsymbol{\sigma}_{v}$ entering (16) and (17) depend on the microstructure of the composite. They are second moments of fields in linear elastic composites and can be expressed by means of classical relations (see Kreher [11] or Ponte Castañeda and Suquet [10] among others). 


\subsection{Derivation of (14)-(17)}

In order to derive (14)-(17), we come back to the local problems (6)-(8) and examine the local fields solutions of these problems in the two limit cases $p=0$ and $p=+\infty$.

- $p=+\infty$ : It follows from (6) that

$$
\lim _{p \rightarrow+\infty} \boldsymbol{L}^{(r)^{*}}(p)=\boldsymbol{L}_{e}^{(r)}
$$

and therefore

$$
\lim _{p \rightarrow+\infty} \boldsymbol{\sigma}^{*}(p)=\boldsymbol{\sigma}_{e}, \quad \lim _{p \rightarrow+\infty} \varepsilon^{*}(p)=\boldsymbol{\varepsilon}_{e}, \quad \lim _{p \rightarrow+\infty} \widetilde{\boldsymbol{L}}^{*}(p)=\widetilde{\boldsymbol{L}}_{e},
$$

where $\sigma_{e}$ and $\varepsilon_{e}$ are the stress and strain fields solutions of the purely elastic problem under a macroscopic strain $\lim _{p \rightarrow+\infty} \bar{\varepsilon}(p)$.

- $p=0$ : Similarly, it follows from (8) that

$$
\lim _{p \rightarrow 0} \mathcal{L}^{(r)^{*}}(p)=\boldsymbol{L}_{v}^{(r)}
$$

and therefore

$$
\lim _{p \rightarrow 0} \boldsymbol{\sigma}^{*}(p)=\boldsymbol{\sigma}_{v}, \quad \lim _{p \rightarrow 0} \varepsilon^{*}(p)=\dot{\boldsymbol{\varepsilon}}_{v}, \quad \lim _{p \rightarrow 0} \widetilde{\mathcal{L}}^{*}(p)=\widetilde{\boldsymbol{L}}_{v},
$$

where $\boldsymbol{\sigma}_{v}$ and $\dot{\boldsymbol{\varepsilon}}_{v}$ are the stress and strain fields solutions of the purely viscous problem. Note that, according to relation (A.6), $\left.\overline{\boldsymbol{\varepsilon}}^{\prime}\right|_{p=0}$ may also be interpreted as the asymptotic macroscopic strain-rate as $t$ tends to $+\infty$.

Relations (14) and (15) result directly from (22) and (20). In order to prove (16), the composite is loaded at constant (in time) macroscopic strain-rate:

$$
\bar{\varepsilon}(t)=\bar{\varepsilon}^{\prime} t
$$

where $\bar{\varepsilon}^{\prime}$ does not depend on $t$. Then

$$
\bar{\varepsilon}^{*}(p)=\frac{1}{p} \bar{\varepsilon}^{\prime} \quad \text { for all } p .
$$

Note that $\boldsymbol{\sigma}^{*}(p)$ and $\boldsymbol{\varepsilon}^{\prime}(p)$ are solution of the "elasticity" problem

$$
\boldsymbol{\sigma}^{*}(p)=\mathcal{L}^{(r)^{*}}(p): \boldsymbol{\varepsilon}^{\prime}(p), \quad \operatorname{div}\left(\boldsymbol{\sigma}^{*}(p)\right)=0, \quad\left\langle\boldsymbol{\varepsilon}^{\prime}(p)\right\rangle=\overline{\boldsymbol{\varepsilon}}^{\prime} .
$$

Consequently:

$$
\overline{\boldsymbol{\varepsilon}}^{\prime}: \widetilde{\mathcal{L}}^{*}(p): \overline{\boldsymbol{\varepsilon}}^{\prime}=\left\langle\boldsymbol{\varepsilon}^{\prime}(p): \mathcal{L}^{*}(p): \boldsymbol{\varepsilon}^{\prime}(p)\right\rangle=\inf _{\boldsymbol{\varepsilon},\langle\boldsymbol{\varepsilon}\rangle=\overline{\boldsymbol{\varepsilon}}^{\prime}}\left\langle\boldsymbol{\varepsilon}: \mathcal{L}^{*}(p): \varepsilon\right\rangle
$$

When the relation (23) is derived with respect to $p$, the result of the derivation reads as (thanks to a lemma given in [10], appendix B, expressing the derivative of a stationary value of an energy):

$$
\overline{\boldsymbol{\varepsilon}}^{\prime}: \frac{\partial \widetilde{\mathcal{L}}^{*}}{\partial p}(p): \overline{\boldsymbol{\varepsilon}}^{\prime}=\left\langle\boldsymbol{\varepsilon}^{\prime}(p): \frac{\partial \mathcal{L}^{*}}{\partial p}(p): \boldsymbol{\varepsilon}^{\prime}(p)\right\rangle .
$$

Taking the derivative with respect to $p$ of the identity

$$
\mathcal{L}^{(r)^{*}}(p): \mathcal{M}^{(r)^{*}}(p)=\boldsymbol{I}, \quad \text { where } \mathcal{M}^{(r)^{*}}(p)=p \boldsymbol{M}_{e}^{(r)}+\boldsymbol{M}_{v}^{(r)},
$$

yields

$$
\frac{\partial \mathcal{M}^{(r)^{*}}}{\partial p}=\boldsymbol{M}_{e}^{(r)}, \quad \frac{\partial \mathcal{L}^{(r)^{*}}}{\partial p}(p)=-\mathcal{L}^{(r)^{*}}(p): \frac{\partial \mathcal{M}^{(r)^{*}}}{\partial p}(p): \mathcal{L}^{(r)^{*}}(p)=-\mathcal{L}^{(r)^{*}}(p): \boldsymbol{M}_{e}^{(r)}: \mathcal{L}^{(r)^{*}}(p)
$$


In particular when $p=0$, making use of relation (22), one gets that:

$$
\frac{\partial \mathcal{L}^{(r)^{*}}}{\partial p}(0)=-\boldsymbol{L}_{v}^{(r)}: \boldsymbol{M}_{e}^{(r)}: \boldsymbol{L}_{v}^{(r)} .
$$

Substituting (26) and (22) into (24) yields (16).

As for (17), note that in a similar way to what was done for $\mathcal{L}^{(r)^{*}}$ :

$$
\frac{\partial \boldsymbol{L}^{(r)^{*}}}{\partial p}(p)=-\boldsymbol{L}^{(r)^{*}}(p): \frac{\partial\left(\boldsymbol{L}^{(r)^{*}}\right)^{-1}}{\partial p}(p): \boldsymbol{L}^{(r)^{*}}(p),
$$

and after due account of the expression (6) of $\boldsymbol{L}^{(r)^{*}}$ :

$$
\frac{\partial \boldsymbol{L}^{(r)^{*}}}{\partial p}(p)=\frac{1}{p^{2}} \boldsymbol{L}^{(r)^{*}}(p): \boldsymbol{M}_{v}^{(r)}: \boldsymbol{L}^{(r)^{*}}(p) .
$$

Therefore

$$
p^{2} \overline{\boldsymbol{\varepsilon}}: \frac{\partial \widetilde{\boldsymbol{L}}^{*}}{\partial p}(p): \overline{\boldsymbol{\varepsilon}}=\left\langle\varepsilon^{*}(p): \boldsymbol{L}^{(r)^{*}}(p): \boldsymbol{M}_{v}^{(r)}: \boldsymbol{L}^{(r)^{*}}(p): \varepsilon^{*}(p)\right\rangle .
$$

Taking the limit of this relation as $p$ tends to $+\infty$, and after accounting for (20), one gets (17).

\subsection{Restrictions on Prony series}

The so-called "collocation method" consists in approximating the actual relaxation function by the Prony series (3), where the relaxation times $\bar{\tau}_{i}$ and the corresponding tensors $\overline{\boldsymbol{L}}_{i}$ have to be chosen, or even optimized, to meet certain requirements (see Turner and Tomé [5], Levesque et al [12], Rekik and Brenner [9]). The approximation (3) is equivalent, after LC transform, to approximating the actual $\widetilde{\boldsymbol{L}}^{*}(p)$ as a sum of rational fractions:

$$
\widetilde{\boldsymbol{L}}^{*}(p) \approx \sum_{i=1}^{M} \frac{p}{p+\frac{1}{\bar{\tau}_{i}}} \overline{\boldsymbol{L}}_{i}, \quad \text { or equivalently } \tilde{\mathcal{L}}^{*}(p) \approx \sum_{i=1}^{M} \frac{1}{p+\frac{1}{\bar{\tau}_{i}}} \overline{\boldsymbol{L}}_{i} .
$$

The four relations (14) to (17) should be imposed to the approximation (27) in order to approach $\widetilde{\boldsymbol{L}}^{*}$ consistently at small and large $t$ 's. The relaxation times $\bar{\tau}_{i}$ and the corresponding weights $\overline{\boldsymbol{L}}_{i}$ must satisfy:

$$
\left.\begin{array}{c}
\sum_{i=1}^{M} \overline{\boldsymbol{L}}_{i} \bar{\tau}_{i}=\widetilde{\boldsymbol{L}}_{v} . \\
\sum_{i=1}^{M} \overline{\boldsymbol{L}}_{i}=\widetilde{\boldsymbol{L}}_{e} . \\
\sum_{i=1}^{M}{\overline{\tau_{i}}}^{2} \overline{\boldsymbol{\varepsilon}}^{\prime}: \overline{\boldsymbol{L}}_{i}: \overline{\boldsymbol{\varepsilon}}^{\prime}=\left\langle\boldsymbol{\sigma}_{v}: \boldsymbol{M}_{e}: \boldsymbol{\sigma}_{v}\right\rangle=\sum_{r=1}^{N} c^{(r)} \boldsymbol{M}_{e}^{(r)}::\left\langle\boldsymbol{\sigma}_{v} \otimes \boldsymbol{\sigma}_{v}\right\rangle^{(r)}, \\
\sum_{i=1}^{M} \frac{1}{\bar{\tau}_{i}} \overline{\boldsymbol{\varepsilon}}: \overline{\boldsymbol{L}}_{i}: \overline{\boldsymbol{\varepsilon}}=\left\langle\boldsymbol{\sigma}_{e}: \boldsymbol{M}_{v}: \boldsymbol{\sigma}_{e}\right\rangle=\sum_{r=1}^{N} c^{(r)} \boldsymbol{M}_{v}^{(r)}::\left\langle\boldsymbol{\sigma}_{e} \otimes \boldsymbol{\sigma}_{e}\right\rangle^{(r)} .
\end{array}\right\}
$$

In particular, these general relations could be imposed as constraints in an optimization procedure similar to the one proposed by Rekik and Brenner [9] to determine the collocation times $\bar{\tau}_{i}$ and the corresponding weights $\overline{\boldsymbol{L}}_{i}$ (note that only the second relation is imposed in [9]). This is left for future work. 
The four above relations are in fact 4 tensorial relations (the last two relations are written as scalar relations but the last two equations hold for arbitrary macroscopic strain $\bar{\varepsilon}$ and for arbitrary macroscopic strain-rate $\bar{\varepsilon}^{\prime}$ ) respectively. These relations provide a systems of $N_{e}$ equations which can be used to determine $N_{e}$ unknowns. $N_{e}$ depends on the symmetry of the tensors $\widetilde{\boldsymbol{L}}$, but it is a multiple of 4 . These equations can be used to determine $N_{e} / 2$ relaxation times $\bar{\tau}_{i}$ 's and the $N_{e} / 2$ corresponding weights $\overline{\boldsymbol{L}}_{i}$ 's from which a Prony series in the form (27) can be constructed. This general principle will be illustrated in section 4 .

\section{Isotropic incompressible composites}

In this section we will give two different examples of the general relations (14)-(17). The first example deals with particle-reinforced composites. The second example addresses the model problem of a checkerboard microstructure. In both cases the individual phases are incompressible. The composite being incompressible and macroscopically isotropic (by assumption), its effective moduli depend on a single shear modulus $\widetilde{\mu}$ (elastic or viscous)

$$
\widetilde{\boldsymbol{L}}=+\infty \boldsymbol{J}+2 \widetilde{\mu} \boldsymbol{K}, \text { or equivalently } \widetilde{\boldsymbol{M}}=\frac{1}{2 \widetilde{\mu}} \boldsymbol{K},
$$

where $\boldsymbol{J}$ and $\boldsymbol{K}$ are the projector on purely hydrostatic and deviatoric symmetric second-order tensors respectively. The form (29) applies to $\widetilde{\boldsymbol{L}}_{e}, \widetilde{\boldsymbol{L}}_{v}, \widetilde{\boldsymbol{L}}^{*}(p)$ and $\overline{\boldsymbol{L}}_{i}$.

Let us examine in more details the form taken by the two last relations in (28) in the two cases of interest.

(i) Two-phase isotropic phases. When the individual constituents are isotropic and incompressible, their elastic or viscous moduli depend on a single shear modulus.

$$
\boldsymbol{M}^{(r)}=\frac{1}{2 \mu^{(r)}} \boldsymbol{K}
$$

where $\boldsymbol{K}$ is the usual projector on purely deviatoric second-order tensors. Each phase has a relaxation time defined as:

$$
\tau^{(r)}=\frac{\mu_{v}^{(r)}}{\mu_{e}^{(r)}}
$$

As is well-known the second moment of the (incompressible) strain field, solution of the linear elastic problem with elastic shear modulus $\mu^{(r)}$ in phase $r$, read as:

Therefore:

$$
\langle\varepsilon: \varepsilon\rangle^{(r)}=\frac{1}{c^{(r)}} \frac{\partial \widetilde{\mu}}{\partial \mu^{(r)}}\left(\mu^{(1)}, \mu^{(2)}\right) \bar{\varepsilon}: \bar{\varepsilon}
$$

$$
\left\langle\boldsymbol{\sigma}_{e}: \boldsymbol{M}_{v}: \boldsymbol{\sigma}_{e}\right\rangle=\sum_{r=1}^{2} c^{(r)} \frac{1}{2 \mu_{v}^{(r)}}\left\langle\boldsymbol{\sigma}_{e}: \boldsymbol{K}: \boldsymbol{\sigma}_{e}\right\rangle^{(r)}=\sum_{r=1}^{2} \frac{2 \mu_{e}^{(r)}}{\tau^{(r)}} \frac{\partial \widetilde{\mu}}{\partial \mu^{(r)}}\left(\mu_{e}^{(1)}, \mu_{e}^{(2)}\right) \bar{\varepsilon}: \bar{\varepsilon} .
$$

Similarly:

$$
\left\langle\boldsymbol{\sigma}_{v}: \boldsymbol{M}_{e}: \boldsymbol{\sigma}_{v}\right\rangle=\sum_{r=1}^{2} c^{(r)} \frac{1}{2 \mu_{e}^{(r)}}\left\langle\boldsymbol{\sigma}_{v}: \boldsymbol{K}: \boldsymbol{\sigma}_{v}\right\rangle^{(r)}=\sum_{r=1}^{2} 2\left(\tau^{(r)}\right)^{2} \mu_{e}^{(r)} \frac{\partial \widetilde{\mu}}{\partial \mu^{(r)}}\left(\mu_{v}^{(1)}, \mu_{v}^{(2)}\right) \overline{\boldsymbol{\varepsilon}}^{\prime}: \overline{\boldsymbol{\varepsilon}}^{\prime} .
$$


(ii) Two-dimensional polycrystals under antiplane shear. The anti-plane problem corresponds to (at least) two different microstructures: a checkerboard microstructure and $2 \mathrm{~d}$ polycrystals made of grains with random orientation (see Bhattacharya and Suquet [13] or Lebensohn et al [14]). The polycrystals are made of cylindrical grains parallel to a given direction $\boldsymbol{e}_{3}$ and their microstructure is therefore two-dimensional (in the plane normal to $\boldsymbol{e}_{3}$ ). Each individual grain is the identical copy, up to a rotation $\theta^{(r)}$, of a reference single crystal having two orthogonal slip systems:

$$
\boldsymbol{m}_{1}=e_{1} \otimes_{s} e_{3}, \quad \boldsymbol{m}_{2}=e_{2} \otimes_{s} e_{3}
$$

where $\boldsymbol{a} \otimes_{s} \boldsymbol{b}$ denotes the symmetric part of the usual tensorial product $\boldsymbol{a} \otimes \boldsymbol{b}$. After rotation, the slip systems in grain $r$ are

$$
\boldsymbol{m}_{1}^{(r)}=\boldsymbol{e}_{1}^{(r)} \otimes_{s} \boldsymbol{e}_{3}, \quad \boldsymbol{m}_{2}^{(r)}=\boldsymbol{e}_{2}^{(r)} \otimes_{s} \boldsymbol{e}_{3},
$$

where $\boldsymbol{e}_{1}^{(r)}$ and $\boldsymbol{e}_{2}^{(r)}$ are rotated from $\boldsymbol{e}_{1}$ and $\boldsymbol{e}_{2}$ by an angle $\theta^{(r)}$. When subjected to anti-plane shear, their deformation is characterized by a scalar displacement along the cylindrical direction $\boldsymbol{e}_{3}$.

The compliance tensor (elastic or viscous) of the single crystal depends on two shear moduli $\mu^{(1)}$ and $\mu^{(2)}$ through:

$$
\boldsymbol{M}^{(r)}=\frac{1}{2 \mu^{(1)}} \boldsymbol{K}_{1}^{(r)}+\frac{1}{2 \mu^{(2)}} \boldsymbol{K}_{2}^{(r)}
$$

where $\boldsymbol{K}_{1}^{(r)}$ and $\boldsymbol{K}_{2}^{(r)}$ are two orthogonal projectors defined as:

$$
\boldsymbol{K}_{1}^{(r)}=\boldsymbol{m}_{1}^{(r)} \otimes \boldsymbol{m}_{1}^{(r)}, \quad \boldsymbol{K}_{2}^{(r)}=\boldsymbol{m}_{2}^{(r)} \otimes \boldsymbol{m}_{2}^{(r)} .
$$

Again, the problem involves two relaxation times, $\tau^{(1)}$ and $\tau^{(2)}$ on each slip system:

$$
\tau^{(r)}=\frac{\mu_{v}^{(r)}}{\mu_{e}^{(r)}}
$$

Classical relations yield certain second moments of the (incompressible) strain and stress fields, solution of the linear elastic problem with shear modulus $\mu^{(1)}$ and $\mu^{(2)}$ :

$$
\left\langle\boldsymbol{\varepsilon}: \boldsymbol{K}_{1}^{(r)}: \boldsymbol{\varepsilon}\right\rangle^{(r)}=\frac{1}{c^{(r)}} \frac{\partial \widetilde{\mu}}{\partial \mu^{(r)}}\left(\mu^{(1)}, \mu^{(2)}\right) \bar{\varepsilon}: \bar{\varepsilon}, \quad\left\langle\boldsymbol{\sigma}: \boldsymbol{K}_{1}^{(r)}: \boldsymbol{\sigma}\right\rangle^{(r)}=\frac{4\left(\mu^{(1)}\right)^{2}}{c^{(r)}} \frac{\partial \widetilde{\mu}}{\partial \mu^{(1)}}\left(\mu^{(1)}, \mu^{(2)}\right) \bar{\varepsilon}: \bar{\varepsilon},
$$

and similar relations with $\boldsymbol{K}_{2}^{(r)}$. These relations yield:

$$
\left\langle\boldsymbol{\sigma}_{e}: \boldsymbol{M}_{v}: \boldsymbol{\sigma}_{e}\right\rangle=\sum_{r=1}^{2} \frac{2 \mu_{e}^{(r)}}{\tau^{(r)}} \frac{\partial \widetilde{\mu}}{\partial \mu^{(r)}}\left(\mu_{e}^{(1)}, \mu_{e}^{(2)}\right) \bar{\varepsilon}: \bar{\varepsilon},
$$

and:

$$
\left\langle\boldsymbol{\sigma}_{v}: \boldsymbol{M}_{e}: \boldsymbol{\sigma}_{v}\right\rangle=\sum_{r=1}^{2} 2\left(\tau^{(r)}\right)^{2} \mu_{e}^{(r)} \frac{\partial \widetilde{\mu}}{\partial \mu^{(r)}}\left(\mu_{v}^{(1)}, \mu_{v}^{(2)}\right) \overline{\boldsymbol{\varepsilon}}^{\prime}: \overline{\boldsymbol{\varepsilon}}^{\prime}
$$

The relations involving second moment of the elastic and viscous stress fields (31) and (32) on one hand, and (34) and (35) are identical. Therefore in both cases the 4 relations (28) take the general form: 


$$
\left.\begin{array}{rl}
\sum_{i=1}^{M} \bar{\mu}_{i} & =\widetilde{\mu}_{e}, \\
\sum_{i=1}^{M} \bar{\tau}_{i} \bar{\mu}_{i} & =\widetilde{\mu}_{v}, \\
\sum_{i=1}^{M} \bar{\mu}_{i} & =\left.\sum_{r=1}^{2} \frac{\mu_{e}^{(r)}}{\bar{\tau}^{(r)}} \frac{\partial \widetilde{\mu}}{\partial \mu^{(r)}}\right|_{e}, \\
\sum_{i=1}^{M} \bar{\mu}_{i} \bar{\tau}_{i}^{2} & =\left.\sum_{r=1}^{2}\left(\tau^{(r)}\right)^{2} \mu_{e}^{(r)} \frac{\partial \widetilde{\mu}}{\partial \mu^{(r)}}\right|_{v}
\end{array}\right\}
$$

where

$$
\widetilde{\mu}_{e}=\widetilde{\mu}\left(\mu_{e}^{(1)}, \mu_{e}^{(2)}\right), \quad \widetilde{\mu}_{v}=\widetilde{\mu}\left(\mu_{v}^{(1)}, \mu_{v}^{(2)}\right)=\widetilde{\mu}\left(\tau^{(1)} \mu_{e}^{(1)}, \tau^{(2)} \mu_{e}^{(2)}\right),
$$

and

$$
\left.\frac{\partial \widetilde{\mu}}{\partial \mu^{(r)}}\right|_{e}=\frac{\partial \widetilde{\mu}}{\partial \mu^{(r)}}\left(\mu_{e}^{(1)}, \mu_{e}^{(2)}\right),\left.\quad \frac{\partial \widetilde{\mu}}{\partial \mu^{(r)}}\right|_{v}=\frac{\partial \widetilde{\mu}}{\partial \mu^{(r)}}\left(\mu_{v}^{(1)}, \mu_{v}^{(2)}\right),=\frac{\partial \widetilde{\mu}}{\partial \mu^{(r)}}\left(\tau^{(1)} \mu_{e}^{(1)}, \tau^{(2)} \mu_{e}^{(2)}\right) .
$$

These 4 equations can be used to identify 4 independent unknowns, namely two relaxation times $\bar{\tau}_{1}$ and $\bar{\tau}_{2}$ and the corresponding weights $\bar{\mu}_{1}$ and $\bar{\mu}_{2}$. The resulting Prony series has only two terms and the corresponding relaxation function and its Laplace transform read as:

$$
\widetilde{\boldsymbol{L}}(t)=+\infty \boldsymbol{J}+2 \widetilde{\mu}(t) \boldsymbol{K}, \quad \widetilde{\mu}(t)=\bar{\mu}_{1} e^{-\frac{t}{\bar{\tau}_{1}}}+\bar{\mu}_{2} e^{-\frac{t}{\bar{\tau}_{2}}}, \quad \widetilde{\mu}^{*}(p)=\frac{p}{p+\frac{1}{\bar{\tau}_{1}}} \bar{\mu}_{1}+\frac{p}{p+\frac{1}{\bar{\tau}_{2}}} \bar{\mu}_{2} .
$$

The model (37) is, in full generality, only an approximation, but on the other hand it improves on the Maxwell model (11) with only one relaxation time.

In order to determine the 4 material parameters $\bar{\tau}_{1}, \bar{\tau}_{2}, \bar{\mu}_{1}$ and $\bar{\mu}_{2}$, the equations (36) are solved in closed form. Upon the change of variables

$$
x_{1}=\frac{\bar{\mu}_{1}}{\widetilde{\mu}_{e}}, \quad x_{2}=\frac{\bar{\mu}_{2}}{\widetilde{\mu}_{e}}, \quad y_{1}=\frac{\bar{\tau}_{1}}{\widetilde{\tau}}, \quad y_{2}=\frac{\bar{\tau}_{2}}{\widetilde{\tau}}, \quad \text { where } \quad \widetilde{\tau}=\frac{\widetilde{\mu}_{v}}{\widetilde{\mu}_{e}} .
$$

the systems (36) becomes:

$$
x_{1}+x_{2}=1, \quad x_{1} y_{1}+x_{2} y_{2}=1, \quad \frac{x_{1}}{y_{1}}+\frac{x_{2}}{y_{2}}=A, \quad x_{1} y_{1}^{2}+x_{2} y_{2}^{2}=B
$$

where

$$
A=\left.\frac{\widetilde{\tau}}{\widetilde{\mu}_{e}} \sum_{r=1}^{2} \frac{\mu_{e}^{(r)}}{\tau^{(r)}} \frac{\partial \widetilde{\mu}}{\partial \mu^{(r)}}\right|_{e}, \quad B=\left.\frac{1}{\widetilde{\mu}_{e} \widetilde{\tau}^{2}} \sum_{r=1}^{2}\left(\tau^{(r)}\right)^{2} \mu_{e}^{(r)} \frac{\partial \widetilde{\mu}}{\partial \mu^{(r)}}\right|_{v}
$$

The solution of (38) is straightforward: after elimination of $x_{1}$ and $x_{2}$ it is found that $y_{1}$ and $y_{2}$ solve the quadratic equation

Noting (see appendix B) that:

$$
y^{2}-\left(B+\frac{B-1}{A-1}\right) y+\frac{B-1}{A-1}=0 .
$$

$$
\left(B+\frac{B-1}{A-1}\right)^{2}-4 \frac{B-1}{A-1} \geq 0
$$

the solution of (38) reads as:

$$
\begin{aligned}
& y_{1} \\
& y_{2}
\end{aligned}=\frac{1}{2}\left(B+\frac{B-1}{A-1}\right) \pm \frac{1}{2} \sqrt{\left(B+\frac{B-1}{A-1}\right)^{2}-4 \frac{B-1}{A-1}},
$$


and

$$
x_{1}=\frac{y_{2}-1}{y_{2}-y_{1}}, \quad x_{2}=\frac{1-y_{1}}{y_{2}-y_{1}} .
$$

Therefore, for each class of microstructure for which an accurate prediction of the effective moduli for linear elastic composites is available in the form of a relation $\left.\left.\widetilde{\mu}\left(\mu^{(1}\right), \mu^{(2}\right)\right)$, the above quantities $A$ and $B$ can be computed and an approximate effective viscoelastic model with two relaxation times, matching exactly the four relations (14) to (17), can be derived for viscoelastic composites.

Note that when the phases are compressible, the same arguments apply separately to the dilatational and deviatoric parts of the constitutive relations. Therefore in addition to the 2 relaxation times (with their corresponding weights) which can be determined for the deviatoric response of compressible composites, 2 other relaxation times (and their corresponding weights) can be determined for their dilatational response.

\subsection{Particle-reinforced two phase composites}

The composites considered in this section are typically made of a matrix reinforced by particles. Both phases are Maxwellian and incompressible. Phase 1 is the matrix whereas the inclusions are considered as phase 2. The effective properties of an isotropic composites made of incompressible linear phases with shear moduli $\mu^{(1)}$ and $\mu^{(2)}$ can be predicted by the Hashin-Shtrikman bound corresponding to the matrix as reference medium:

$$
\widetilde{\mu}\left(\mu^{(1)}, \mu^{(2)}\right)=\mu^{(1)}+c^{(2)} \frac{\mu^{(2)}-\mu^{(1)}}{1+c^{(1)} \beta \frac{\mu^{(2)}-\mu^{(1)}}{\mu^{(1)}}}, \quad \beta=\frac{2}{2+d} \quad d=2 \text { or } 3 \text { (dimension of space). }
$$

Then:

$$
\frac{\partial \widetilde{\mu}}{\partial \mu^{(2)}}=\frac{c^{(2)}}{\left(1+c^{(1)} \beta \frac{\mu^{(2)}-\mu^{(1)}}{\mu^{(1)}}\right)^{2}}, \quad \frac{\partial \widetilde{\mu}}{\partial \mu^{(1)}}=\frac{1}{\mu^{(1)}}\left(\widetilde{\mu}-\mu^{(2)} \frac{\partial \widetilde{\mu}}{\partial \mu^{(2)}}\right) .
$$

The above relations (43) and (44) allow to derive explicit expressions for $A$ and $B$ in (38). The solution to $(38)$ then reads as:

$$
\left.\begin{array}{c}
\bar{\tau}_{1}=\tau^{(1)}, \quad \bar{\tau}_{2}=\tau^{(1)} \tau^{(2)} \frac{\mu_{e}^{(1)}\left(1-\beta c^{(1)}\right)+\mu_{e}^{(2)} \beta c^{(1)}}{\tau^{(1)} \mu_{e}^{(1)}\left(1-\beta c^{(1)}\right)+\tau^{(2)} \mu_{e}^{(2)} \beta c^{(1)}}, \\
\bar{\mu}_{1}=\mu_{e}^{(1)} \frac{c^{(1)}(1-\beta)}{1-\beta c^{(1)}}, \quad \bar{\mu}_{2}=\mu_{e}^{(1)} \mu_{e}^{(2)} \frac{1-c^{(1)}}{\left(1-\beta c^{(1)}\right)} \frac{1}{\mu_{e}^{(1)}\left(1-\beta c^{(1)}\right)+\mu_{e}^{(2)} \beta c^{(1)}} .
\end{array}\right\}
$$

Rougier [6], Ricaud and Masson [8] have shown that when the microstructure of the composite is such that its effective elastic properties are accurately described by the Hashin-Shtrikman bound, the exact relaxation function is the sum of two exponentials as in (37). The relaxation times and the corresponding weights that they have derived coincide with (45). Therefore, in this particular case the approximate model (37) is exact.

\subsection{2d polycrystals under anti-plane shear}

The exact effective antiplane elastic and viscous shear modulus of such a composite are given (classically) as:

$$
\widetilde{\mu}_{e}=\sqrt{\mu_{e}^{(1)} \mu_{e}^{(2)}}, \quad \widetilde{\mu}_{v}=\sqrt{\mu_{v}^{(1)} \mu_{v}^{(2)}}, \quad \widetilde{\tau}=\sqrt{\tau^{(1)} \tau^{(2)}} .
$$


The exact effective relaxation function of the composite cannot be expressed as a Prony series (see Rougier et $a l$ [6] and Beurthey and Zaoui [15] for a similar result for two-phase isotropic 3d polycrystals). The equations (36) become:

$$
\left.\begin{array}{c}
\bar{\mu}_{1}+\bar{\mu}_{2}=\widetilde{\mu}_{e}, \quad \bar{\mu}_{1} \bar{\tau}_{1}+\bar{\mu}_{2} \bar{\tau}_{2}=\widetilde{\mu}_{e} \widetilde{\tau} \\
\bar{\mu}_{1} \frac{1}{\bar{\tau}_{1}}+\bar{\mu}_{2} \frac{1}{\bar{\tau}_{2}}=\frac{1}{2} \widetilde{\mu}_{e}\left(\frac{1}{\tau^{(1)}}+\frac{1}{\tau^{(2)}}\right), \quad \bar{\mu}_{1} \bar{\tau}_{1}^{2}+\bar{\mu}_{2} \bar{\tau}_{2}^{2}=\frac{1}{2} \widetilde{\mu}_{e} \widetilde{\tau}\left(\tau^{(1)}+\tau^{(2)}\right)
\end{array}\right\}
$$

and using the general form (41) and (42) of the solution, it is found that

$$
\left.\begin{array}{c}
\bar{\tau}_{1}=\frac{1}{4}\left(\sqrt{\tau^{(1)}}+\sqrt{\tau^{(2)}}\right)^{2}+\frac{1}{2 \sqrt{2}}\left(\sqrt{\tau^{(1)}}-\sqrt{\tau^{(2)}}\right) \sqrt{\frac{1}{2}\left(\sqrt{\tau^{(1)}}+\sqrt{\tau^{(2)}}\right)^{2}+2 \widetilde{\tau}} \\
\bar{\tau}_{2}=\frac{1}{4}\left(\sqrt{\tau^{(1)}}+\sqrt{\tau^{(2)}}\right)^{2}-\frac{1}{2 \sqrt{2}}\left(\sqrt{\tau^{(1)}}-\sqrt{\tau^{(2)}}\right) \sqrt{\frac{1}{2}\left(\sqrt{\tau^{(1)}}+\sqrt{\left.\tau^{(2)}\right)^{2}+2 \widetilde{\tau}}\right.} \\
\bar{\mu}_{1}=\widetilde{\mu}_{e} \frac{\bar{\tau}_{2}}{\bar{\tau}_{2}+\widetilde{\tau}}=\widetilde{\mu}_{e} \frac{\sqrt{\bar{\tau}_{2}}}{\sqrt{\bar{\tau}_{1}}+\sqrt{\bar{\tau}_{2}}} \\
\bar{\mu}_{2}=\widetilde{\mu}_{e} \frac{\bar{\tau}_{1}}{\bar{\tau}_{1}+\widetilde{\tau}}=\widetilde{\mu}_{e} \frac{\sqrt{\bar{\tau}_{1}}}{\sqrt{\bar{\tau}_{1}}+\sqrt{\bar{\tau}_{2}}}
\end{array}\right\}
$$

In order to check the accuracy of the model (37) with two relaxation times, the composite is subjected to an antiplane shear loading history consisting of two different stages. In a first stage the shear deformation is increased at constant strain-rate $\dot{\varepsilon}_{0}$ between 0 and $t_{0}$. In a second stage the composite is subjected to a cyclic deformation at frequency $\omega$ :

$$
\bar{\varepsilon}_{13}(t)=\dot{\varepsilon}_{0} t \quad t \leq t_{0}, \quad \bar{\varepsilon}_{13}(t)=\dot{\varepsilon}_{0} t_{0}+\frac{\dot{\varepsilon}_{0}}{\omega} \sin \left(\omega\left(t-t_{0}\right)\right) \quad t \geq t_{0}, \quad \bar{\varepsilon}_{23}(t)=0 .
$$

The predictions of (37), which can be obtained in closed form making use of the relations given in appendix $\mathrm{C}$, are compared with a full-field simulation performed with a computational method based on Fast Fourier Transforms (introduced in Moulinec and Suquet [16,17] and used in particular in Bhattacharya and Suquet [13] and Lebensohn et al [14]). The predictions of the effective Maxwell model (11) are also given for comparison. Two sets of data have been used, the first one corresponding to a moderate contrast between the relxation times of the individual phases $\tau^{(1)} / \tau^{(2)}=10$, the second one corresponding to a stronger contrast $\tau^{(1)} / \tau^{(2)}=100$.

$$
\left.\begin{array}{cl}
\text { Moderate contrast: } & \mu_{e}^{(1)}=1 \mathrm{MPa}, \mu_{v}^{(1)}=2 \mathrm{MPa} . \mathrm{s}, \tau^{(1)}=2 \mathrm{~s}, \\
& \mu_{e}^{(2)}=100 \mathrm{MPa}, \mu_{v}^{(1)}=20 \mathrm{MPa} . \mathrm{s}, \tau^{(2)}=0.2 \mathrm{~s} .
\end{array}\right\}
$$

The predictions of the models are compared with the full-field simulations in figure 1. As expected both models capture correctly the initial slope (elastic response) and the asymptotic response of the composite. However, even at moderate contrast, the transient response of the composite is not well approximated by the Maxwell model whereas the predictions of the model with two relaxation times are in good agreement with the FFT simulations. They deviate from them when the contrast is strong. This observation is consistent with the fact that the exact relaxation function for this problem is a continuous function with support between $\tau^{(1)}$ and $\tau^{(2)}$ and not two Dirac masses. 

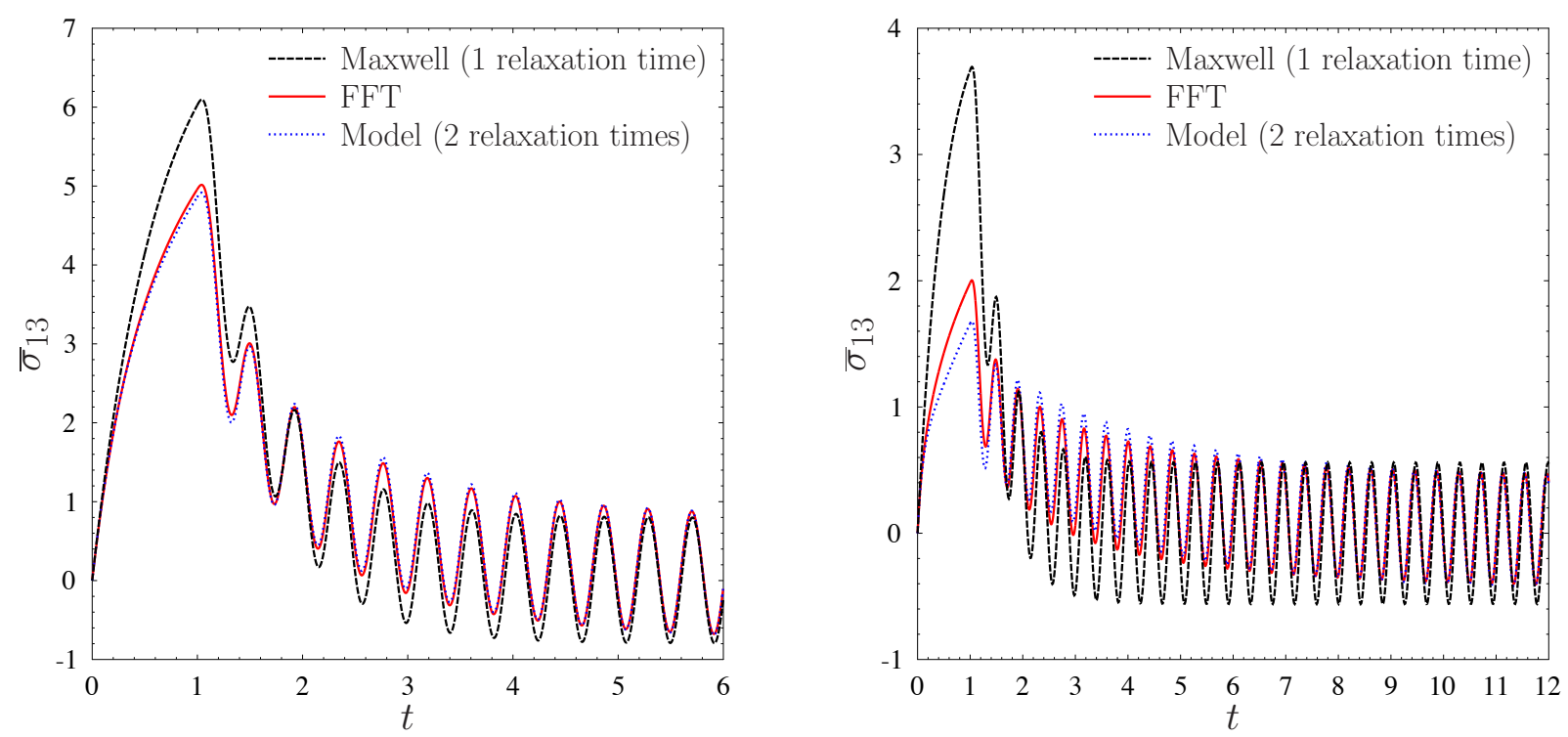

Figure 1. Antiplane problem for a checkerboard. Comparison between a full-field simulation (FFT), the Maxwell model (11) with a single relaxation time and with the model (37) with two relaxation times. Left: data (49) corresponding to a moderate contrast between the relaxation times of the phases. Right: data (50) corresponding to a strong contrast between the relaxation times of the phases.

\section{Concluding remarks.}

Four asymptotic relations for the effective relaxation function of linear viscoelastic composites have been derived. These relations require the resolution of linear elastic (or purely viscous) problems. Besides their intrinsic interest, these four relations can be used in (at least) two ways:

- They can be used as constraints for the identification of collocation times (and the corresponding weights) within a collocation method.

- The relations provide four equations which can be used to determine (at least) two relaxation times with their corresponding weights.

The second direction has been briefly investigated here for incompressible constituents and the accuracy of the resulting approximate model has been discussed.

These relations were established here for the relaxation function of composites with Maxwellian constituents. Similar relations exist for the creep functions of these composites and of composites with Kelvin-Voigt constituents.

\section{Acknowledgements}

This study was partly funded by the French 'Agence Nationale de la Recherche' (project ELVIS, \#ANR-08-BLAN-0138). The author is grateful to H. Moulinec (LMA-CNRS, France) for providing the FFT simulations shown in figure 1. Fruitful discussions with R. Brenner are also gratefully acknowledged. 


\section{References}

[1] J. Sanchez-Hubert, E. Sanchez-Palencia, Sur certains problèmes physiques d'homogénéisation donnant lieu à des phénomènes de relaxation, C.R. Acad. Sc. Paris Série A 286 (1978) 903-906.

[2] N. Laws, R. Mc Laughlin, Self-consistent estimates for the viscoelastic creep compliance of composite materials, Proc. R. Soc. London A 359 (1978) 251-273.

[3] P. Suquet, Elements of Homogenization for Inelastic Solid Mechanics, in: E. Sanchez-Palencia, A. Zaoui (Eds.), Homogenization Techniques for Composite Media, Vol. 272 of Lecture Notes in Physics, Springer Verlag, New York, 1987, pp. 193-278.

[4] G. Francfort, P. Suquet, Homogenization and mechanical dissipation in thermo-viscoelasticity, Arch. Rat. Mech. Anal. 96 (1986) 265-293.

[5] P. Turner, C. Tomé, Self-consistent modeling of visco-elastic polycrystals: application to irradiation creep and growth, J. Mech. Phys. Solids 41 (1993) 1191-1211.

[6] Y. Rougier, C. Stolz, A. Zaoui, Représentation spectrale en viscoélasticité linéaire des matériaux hétérogènes, C.R. Acad. Sc. Paris, II 316 (1993) 1517-1522.

[7] Y. Rougier, Etude du comportement sous irradiation: modélisation micromécanique de l'élastoviscoplasticité., Ph.D. thesis, Ecole Polytechnique, France (1994).

[8] J. Ricaud, R. Masson, Effective properties of linear viscoelastic heterogeneous media: Internal variables formulation and extension to ageing behaviours, Int. J. Solids Structures 46 (2009) 1599-1606.

[9] A. Rekik, R. Brenner, Optimization of the collocation inversion method for the linear viscoelastic homogenization, Mechanics Research Communications 38 (2011) 305-308.

[10] P. Ponte Castañeda, P. Suquet, Nonlinear composites, in: E. V. der Giessen, T. Wu (Eds.), Advances in Applied Mechanics, Vol. 34, Academic Press, New York, 1998, pp. 171-302.

[11] W. Kreher, Residual stresses and stored elastic energy of composites and polycrystals, J. Mech. Phys. Solids 38 (1990) 115-128.

[12] M. Lèvesque, M. Gilchrist, N. Bouleau, K. Derrien, D. Baptiste, Numerical inversion of the Laplace-Carson transform applied to homogenization of randomly reinforced linear viscoelastic media, Comput. Mech. 40 (2007) 771-789.

[13] K. Bhattacharya, P. Suquet, A model problem concerning recoverable strains of shape-memory polycrystals, Proc. R. Soc. London A 461 (2005) 2797-2816.

[14] R. Lebensohn, R. Brenner, O. Castelnau, P. Gilormini, Study of the antiplane deformation of linear 2-d polycrystals with different microstructures, Int. J. Solids Structures 42 (2005) 5441-5459.

[15] S. Beurthey, A. Zaoui, Structural morphology and relaxation spectra of viscoelastic heterogeneous materials, Eur. J. Mech. A/Solids 19 (2000) 1-16.

[16] H. Moulinec, P. Suquet, A fast numerical method for computing the linear and nonlinear properties of composites, C. R. Acad. Sc. Paris II 318 (1994) 1417-1423.

[17] H. Moulinec, P. Suquet, A numerical method for computing the overall response of nonlinear composites with complex microstructure, Comp. Meth. Appl. Mech. Engng. 157 (1998) 69-94.

[18] Q. H. Vu, R. Brenner, O. Castelnau, H. Moulinec, P. Suquet, A self-consistent estimate for linear viscoelastic polycrystals with internal variables inferred from the collocation method, Modelling and Simulation in Materials Science and Engineering, submitted.

\section{Appendix A. Laplace and Laplace-Carson transforms}

The Laplace transform $\mathcal{L} f$ and the Laplace-Carson transform $f^{*}(p)$ of a function of time $f$ are defined as

$$
\mathcal{L} f=\int_{0}^{+\infty} e^{-p t} f(t) d t, \quad f^{*}(p)=p \int_{0}^{+\infty} e^{-p t} f(t) d t=p \mathcal{L} f
$$


The following elementary properties of the Laplace-Carson transform are worth noting (similar relations exist for the Laplace transform):

1) Derivation:

$$
\mathcal{L} \dot{f}(p)=p \mathcal{L} f(p)-f(0), \quad \dot{f}^{*}(p)=p\left(f^{*}(p)-f(0)\right),
$$

2) Riemann and Stieljes convolution:

$$
\begin{aligned}
& \text { Riemann : }(f \star g)(t)=\int_{0}^{t} f(t-s) g(s) d s, \quad \mathcal{L}(f \star g)(p)=\mathcal{L} f(p) \mathcal{L} g(p), \\
& \text { Stieljes : }(f \otimes g)(t)=\frac{d}{d t}(f \star g)(t)=f(t) g(0)+\int_{0}^{t} f(t-s) \dot{g}(s) d s, \\
&(f \otimes g)^{*}(p)=f^{*}(p) g^{*}(p),
\end{aligned}
$$

3) Short time behavior:

4) Long time behavior:

$$
\lim _{p \rightarrow+\infty} f^{*}(p)=\lim _{t \rightarrow 0^{+}} f(t)
$$

$$
\begin{aligned}
\lim _{p \rightarrow 0} f^{*}(p) & =\lim _{t \rightarrow+\infty} f(t), \\
\lim _{p \rightarrow 0} p f^{*}(p) & =\lim _{t \rightarrow+\infty} \frac{f(t)}{t},
\end{aligned}
$$

\section{Appendix B. Proof of (40)}

It is first shown that:

$$
B \geq 1 .
$$

To prove (B.1), first note that:

$$
\left\langle\boldsymbol{\sigma}_{v}: \boldsymbol{M}_{e}: \boldsymbol{\sigma}_{v}\right\rangle \geq \overline{\boldsymbol{\sigma}}_{v}: \widetilde{\boldsymbol{M}}_{e}: \overline{\boldsymbol{\sigma}}_{v}, \quad \text { where } \quad \overline{\boldsymbol{\sigma}}_{v}=\left\langle\boldsymbol{\sigma}_{v}\right\rangle .
$$

Taking into account the overall isotropy and incompressibility of the composite, it is found that

and therefore

$$
\overline{\boldsymbol{\sigma}}_{v}: \widetilde{\boldsymbol{M}}_{e}: \overline{\boldsymbol{\sigma}}_{v}=\frac{2 \widetilde{\mu}_{v}^{2}}{\widetilde{\mu}_{e}} \overline{\boldsymbol{\varepsilon}}^{\prime}: \overline{\boldsymbol{\varepsilon}}^{\prime},
$$

In a second step, it is observed that:

$$
B=\frac{1}{2} \frac{1}{\widetilde{\mu}_{e} \widetilde{\tau}^{2}} \frac{1}{\bar{\varepsilon}^{\prime}: \bar{\varepsilon}^{\prime}}\left\langle\boldsymbol{\sigma}_{v}: \boldsymbol{M}_{e}: \boldsymbol{\sigma}_{v}\right\rangle \geq 1 .
$$

$$
\left(B+\frac{B-1}{A-1}\right)^{2}-4 \frac{B-1}{A-1} \geq \inf _{x}\left(B^{2}+2 B x+x^{2}-4 x\right),
$$

where $x$ stands for $(B-1) /(A-1)$. The above infimum is $4(B-1)$ and according to the first step, it is positive. This completes the proof of (40).

\section{Appendix C. Alternative writing of the constitutive equations under the approximation (27)}

It is worth noting, along the lines of Ricaud and Masson [8], that the constitutive relations corresponding to the approximation based on Prony series may be more conveniently expressed by the introduction 
of internal variables (a similar observation is made in [18]). Indeed, under approximation (27), the constitutive relations read in Laplace space as:

$$
\overline{\boldsymbol{\sigma}}^{*}(p)=\sum_{i=1}^{M} \frac{p}{p+\frac{1}{\bar{\tau}_{i}}} \overline{\boldsymbol{L}}_{i}: \overline{\boldsymbol{\varepsilon}}^{*}(p)=\sum_{i=1}^{M} \overline{\boldsymbol{L}}_{i}:\left(\overline{\boldsymbol{\varepsilon}}^{*}(p)-\boldsymbol{\beta}_{i}^{*}(p)\right)
$$

with

$$
\boldsymbol{\beta}_{i}^{*}(p)=\frac{1}{\bar{\tau}_{i} p+1} \bar{\varepsilon}^{*}(p) .
$$

Taking the inverse LC transform of (C.1) and (C.2) yields

$$
\overline{\boldsymbol{\sigma}}(t)=\sum_{i=1}^{M} \overline{\boldsymbol{L}}_{i}:\left(\overline{\boldsymbol{\varepsilon}}(t)-\boldsymbol{\beta}_{i}(t)\right)
$$

with

$$
\bar{\tau}_{i} \dot{\boldsymbol{\beta}}_{i}(t)+\boldsymbol{\beta}_{i}(t)=\overline{\boldsymbol{\varepsilon}}(t), \quad \beta_{i}(0)=0 .
$$

The model (C.3)(C.4) is a generalized Maxwell model consisting $M$ Maxwell rheological elements assembled in parallel. The $i$-th element has stiffness $\overline{\boldsymbol{L}}_{i}$ and viscosity $\bar{\tau}_{i} \overline{\boldsymbol{L}}_{i}$ and $\boldsymbol{\beta}_{i}$ is the viscous strain in this element.

The differential equation

$$
\tau \dot{\boldsymbol{\beta}}+\boldsymbol{\beta}=\overline{\boldsymbol{\varepsilon}}(t),
$$

can be integrated explicitely:

$$
\boldsymbol{\beta}(t)=\int_{0}^{t} e^{\frac{s-t}{\tau}} \overline{\boldsymbol{\varepsilon}}(s) d s .
$$

For instance, under the loading (48), the explicit solution of (C.4) reads as:

$$
\begin{aligned}
& \text { Phase } 1: t \leq t_{0} \quad \beta(t)=\dot{\varepsilon}_{0}\left(t-\tau+\tau e^{-\frac{t}{\tau}}\right), \quad \dot{\beta}(t)=\dot{\varepsilon}_{0}\left(1-e^{-\frac{t}{\tau}}\right) . \\
& \text { Phase } 2: t \geq t_{0}\left\{\begin{aligned}
\beta(t) & =\beta\left(t_{0}\right)-\tau\left(\dot{\beta}\left(t_{0}\right)-\frac{\dot{\varepsilon}_{0}}{1+\tau^{2} \omega^{2}}\right)\left(e^{\frac{t_{0}-t}{\tau}}-1\right) \\
& +\frac{\dot{\varepsilon}_{0}}{1+\tau^{2} \omega^{2}}\left[\frac{1}{\omega} \sin \left(\omega\left(t-t_{0}\right)\right)-\tau\left(\cos \left(\omega\left(t-t_{0}\right)-1\right)\right]\right. \\
\dot{\beta}(t) & =\left(\dot{\beta}\left(t_{0}\right)-\frac{\dot{\varepsilon}_{0}}{1+\tau^{2} \omega^{2}}\right) e^{\frac{t_{0}-t}{\tau}} \\
& +\frac{\dot{\varepsilon}_{0}}{1+\tau^{2} \omega^{2}}\left[\cos \left(\omega\left(t-t_{0}\right)\right)+\tau \omega \sin \left(\omega\left(t-t_{0}\right)\right]\right.
\end{aligned}\right.
\end{aligned}
$$

where

$$
\beta\left(t_{0}\right)=\dot{\varepsilon}_{0}\left(t_{0}-\tau+\tau e^{-\frac{t_{0}}{\tau}}\right), \quad \dot{\beta}\left(t_{0}\right)=\dot{\varepsilon}_{0}\left(1-e^{-\frac{t_{0}}{\tau}}\right) .
$$

